\title{
社区网络的使用及其对社区参与的影响 以南京市为例
}

\author{
常恩予 ${ }^{1}$, 甄 峰 ${ }^{1,2^{*}}$, 孙 晨 ${ }^{1}$
}

(1. 南京大学建筑与城市规划学院, 南京 210093;2. 南京大学人文地理研究中心, 南京 210093)

\begin{abstract}
摘 要: 20 世纪 70 年代,美国在社区信息化建设中开展始了社区内部信息平台“社区网络(community network)”的 大量实践和研究。近年来随着信息通讯技术的快速发展, 中国城市社区基于实体社区的虚拟网络平台也大量兴 起, 为改善城市社区参与不足的现状提供了契机。目前国内学者对社区网络的关注不足, 相关定量研究更少。本 文结合中国社区网络实践, 以南京市为例, 综合考虑区位、交通等因素, 选取主城区 35 个社区进行问卷调研, 根据 调研结果对城市社区居民社区网络的使用情况及社区参与的线上线下特征进行分析, 并从“媒介类型”和“用途类 型”2 个维度就社区网络对社区参与的影响进行回归分析。研究表明:南京市城市居民社区网络使用总体呈现“高 普及、低强度、新社区”的特征, 社区网络的普及度较高, 然而平均使用强度并不高; 在社区网络类型的使用方面呈 现 “以移动互联为主”的特征, 居民使用度最高的是社区 $\mathrm{QQ}$ 群/微信群, 其次为社区公众号/APP, 使用度最低的为 社区网站/社区论坛; 在社区“O2O”方面, 城市居民社区网络的使用对线下社区参与也有显著促进作用, 且社区网 络的使用有助于社区参与深度的提升, 促进非事务型社区参与向事务型社区参与的演进。未来智慧社区建设应弥 补对社区网络及社区参与关注的不足, 积极引导居民通过线上重新回到线下, 通过社区活动的参与融人社区公共 生活, 从而激发社区活力。
\end{abstract}

关 键 词: 城市社区; 社区参与; 社区网络; 智慧社区; 社区“ $O 2 O$ ”; 南京

\section{1 引言}

社区的概念形成之初, 滕尼斯等学者所关注的 传统社区的内涵为“建立在血缘、地缘、情感和自然 意志之上的富有人情味和认同感的传统社会生活 共同体” (滕尼斯, 1999), 社区内部成员的互动以及 对共同利益的关注是社区形成中不可忽视的重要 因素, 居民对各类事务型和非事务型的社区活动的 参与为重要环节(张庭伟, 1999; 叶南客, 2001; 吴光 芸等, 2006)。随着现代化进程的不断推进, 城市居 民日益远离社区公共生活, 社区参与减弱; 西方社 会在二战后的一段时期也出现了社区衰落和公共 参与递减的现象(吴晓林等, 2015)。社区参与是社 会资本中 “良性循环” 的核心环节, 是协商和解决社
区公共事务的重要平台, 也是社区满意度的重要影 响因素。在此背景下,许多学者开展了社区参与的 影响因素相关研究,试图找到重新激发社区活力的 良药。一些学者将社区归属感定义为社区参与的 “催化剂”, 对社区归属感与各种社区参与行为之间 的关联进行验证。研究表明,个人对社区的认同和 归属感愈强烈, 便愈有可能加人社区组织, 进行社 区参与(Chavis et al, 1990; Brodsky et al, 1999; Anderson, 2009)。Putnam(2000)有关社会资本的研究 表明, 非政治性参与有助于建立信任感和社会网 络, 从而促进政治性参与。Xu等(2010)验证了邻里 交往对社区参与的促进作用。另外,大量的研究表 明,社会经济属性与社区集体有效解决社区事务的 信任度相关(Carroll, Reese, 2003), 年龄和家庭结构

收稿日期: 2016-08; 修订日期: 2017-02。

基金项目: 国家自然科学基金项目(41571146)[Foundation: National Natural Science Foundation of China, No.41571146]。 作者简介: 常恩予(1991-),女, 山东济宁人,硕士研究生,主要研究方向为智慧社区、社区参与,E-mail: choloncey@126.com。 通讯作者: 甄峰(1973-), 男, 陕西汉中人, 教授, 博士生导师, 主要从事信息时代城市与区域空间结构、智慧城市等方面研究, E-mail: zhenfeng@nju.edu.cn。

引用格式: 常恩予, 甄峰, 孙晨. 2017. 社区网络的使用及其对社区参与的影响: 以南京市为例[J]. 地理科学进展, 36(7): 785-794. [Chang E Y, Zhen F, Sun C. 2017. Use of community networks and its impact on community participation: A case study of Nanjing City[J]. Progress in Geography, 36(7): 785-794.]. DOI: 10.18306/dlkxjz.2017.07.001 
也与社区参与类型相关, 老年人更乐于进行政治参 与, 有 18 岁以下孩子的家庭更乐于进行教育类的活 动参与 (Milbrath, 1977)。此外, 为突破中国社区参 与不足的困境, 国内一些学者就中国特有的制度性 和社会性因素对社区参与的影响进行了研究。杨 敏(2007)的研究表明, 各参与类型的性质和具体参 与过程不仅取决于居民自身的社会资源和行动能 力, 还受到国家权力和社区建设导向的决定性影 响。陈振华(2004)、孙璐(2006)等学者认为, 长期以 来社区功能的萎缩, 制度供给不足已经成为制约居 民社区参与的主要因素, 这种制度供给不足表现在 居委会的行政化、业主委员会的职能单一和社区自 治组织缺乏等方面。

随着信息技术的飞速发展和向社会公共领域 的不断渗透, 20 世纪末起, 西方学者越来越多的关 注互联网对于公众参与、社区参与的影响, 虽然有 学者认为互联网降低了参与的成本, 为参与提供了 更加充足的信息和更加便利的途径(Bimber, 1998; Dimaggio et al, 2003); 但也有许多研究表明, 互联网 的使用在某种程度上减弱了社区参与的积极性, 因 为互联网最大的特色在于它超越距离, 因而很多网 络活动是以牺牲本地化和面对面交往的时间为代 价, 且网络主要用于娱乐休闲, 降低了人的社会性, 居民投人到公共生活中的时间将会更少(Putnam, 1995; Wellman et al, 2001)。“社区网络(Community Network)” 的出现为改善这一现状带来了新的转 机。社区网络是基于实体社区的虚拟网络平台, 用 于社区信息的发布和管理、邻里交流和互动, 以及 社区活动和社区服务等方面(Carroll, Rosson, 2003; Kavanaugh, 2005a)。这种虚拟社区和实体社区合 而为一的媒介, 将同一地域范围的社区居民粘连在 同一个虚拟空间中, 它的出现, 为社区“ $\mathrm{O} 2 \mathrm{O}$ ” (Online to Offline/Offline to Online, 线上线下融合)的实 现带来了充分的可能, 也为改善社区参与不足的现 状提供了契机。

社区网络并不是近期出现的新概念, 早在 20 世 纪 70年代, 美国的社区信息化建设中就开展了社区 信息平台的建设实践(Schuler, 1996), 促进社会活动 是其重要的实践领域之一。最初社区网络的形式 以远程登录(Telnet)和电子邮件的形式为主, 至 1990 年代中期, 发展为社区网站形式。如今, 随着互联 网的广泛普及和应用, 社区网络的形式又趋向多样 化, 资源的接人也更加丰富、便捷。西方的大量研 究表明,社区网络的使用可有效地促进社区参与。
有学者通过对一个成熟的网络化社区的长时间跟 踪研究, 证明了社区网络的使用会增强社区参与, 并且在某种程度上受年龄、受教育程度以及性格外 向度等因素的影响(Kavanaugh et al, 2005b)。Keith 的研究表明,社区网络作为一种组织媒介, 通过加 强社区内部的的社会联系而促进社区集体活动(引 自 Hampton, 2003)。还有研究认为, 作为诸多互动 的支撑, 社区网络的意义超越了一个媒介, 而成为 同社区组织、社区成员共同组成的行动者之一, 成 为一个持久的社区社会结构 (Kling et al, 1989)。随 着信息技术的发展, 社区网络的形态也发生转变, 因此其作用也需不断地持续研究。

近年来, 中国在社区网络方面也涌现出大量实 践。在信息和通讯技术逐步深人居民生活的背景 下,受互联网企业与线下服务结合、社区管理意识 转型、服务意识增强等因素的影响,不论是在政府 导向的智慧社区建设实践和市场导向的物业公司 服务升级, 还是居民的自发组织中, 都相继开启了 社区网络平台的建设热潮。现阶段主要有即时交 流群(社区 QQ群/微信群)、网页(如社区网站/论坛) 和服务终端(社区公众号/APP)等3种类型。相比之 下, 在社区参与和智慧社区的研究领域, 目前国内 学者对社区网络的应用关注不足, 仅有部分相关研 究对如何利用社区平台促进社区互动的相关思路 和策略进行了介绍(申悦等, 2014; 柴彦威等, 2015; 甄峰等, 2015), 少量定量研究对信息技术的使用对 于社区居民的邻里交往以及社区满意度等方面的 影响进行了关注(陈卉等, 2016)。而在社区内部的 网络平台与社区参与的关系方面, 较为深人的定量 研究则是少之又少。那么,社区网络在中国社区居 民中的使用情况如何, 对城市社区居民的社区参与 又会产生怎样的影响, 不同的社区网络类型对于社 区参与的影响又有何不同? 在此背景之下, 本文对 南京市城市居民社区网络的使用及社区参与的线 上线下特征进行分析,并从“媒介类型”和“用途类 型”2个维度就社区网络对社区参与的影响进行分 析,进而探讨信息社会背景下促进社区参与的新路 径, 为智慧社区建设提供科学指引。

\section{2 数据来源与研究方法}

\section{1 数据来源}

本文以居住小区作为社区的实际界定单元, 因 为居住小区是一个可以从心理、经济与地理等方面 
清晰分辨边界的邻里(黄荣贵等, 2011)。研究数据 来源于南京市社区问卷调研, 调研时间为 2015 年 12 月-2016年 4 月。此次调研综合考虑区位、交通 等因素, 选择南京市主城区共 35 个社区, 调研社区 包含 4 种类型, 分别为新型商品房社区, 老旧商品房 社区, 单位型社区和保障房社区。各类社区的问卷 发放总数量按照南京市住房类型规模比例确定分 层抽样数量, 每个社区发放数量按照人口总户数抽 样。各调研社区在空间上均匀分布于南京市主城 8 个行政区, 研究组成员深人社区内部随机抽样进行 人户调研, 对 18 岁以上的社区居民进行问卷发放和 访谈,调研时间包含工作日的下班时间和周末。问 卷主要包括社区参与、邻里交往、社区服务、社区满 意度以及社区网络使用情况和个人社会经济属性 等方面的内容。本次调研共回收问卷 1600 份, 有效 问卷 1560 份, 问卷有效率为 $97.5 \%$ 。其中调研样本 的人口年龄结构与南京市 2015 年 $1 \%$ 人口抽样调查 中的年龄结构基本一致。根据研究目的, 本文选取 社区网络和社区参与部分数据完整的 1258 个样本 (由于篮选后的少量样本仍存在个别变量缺失, 在 模型分析时进行了均值插补处理, 以保证模型分析 的有效性)用于分析。调研社区分布情况如图 1, 部 分样本统计描述如表 1 。

\section{2 研究方法}

第一步, 各变量的测度。首先, 对因变量社区 参与的测度进行规定。社区居民参与社区公共生 活的方式有多种, 根据国内外许多研究对社区参与 形式的总结, 从参与的深度可分为浅度参与和深度
参与,浅度参与包括问题的关注、信息的搜集与讨 论, 深度参与包括与公共事务进行直接接触, 包括 实际参与选举等等(Almond et al, 1963); 从参与的 内容的性质可以划分为社区政治参与、社区经济参 与、社区文化参与、社区社会参与等方面(王刚等, 1999; 张卫, 2001; 陈雅丽, 2002)。综合以往研究成 果, 本文分别从 “事务型参与、志愿型参与、文教型 参与、休闲型参与” 4 个维度和 “信息查找、参与活 动、参与组织” 3 个深度进行社区参与度的分析和测 度。其次, 对解释变量的测度进行规定。社区网络 的使用是本文最主要的解释变量, 从社区网络的 “媒介类型”和“用途类型” 2 个维度人手进行测度， 其中社区网络的“媒介类型”包括社区论坛/网站、 QQ群/微信群、公众号/APP等 3 种, 与社区参与的 维度相匹配,社区网络的“用途类型”分为“公共事 务类、公益志愿类、文化教育类、休闲娱乐类”。另 外,控制变量包括被访者性别(女性为参照项)、年龄

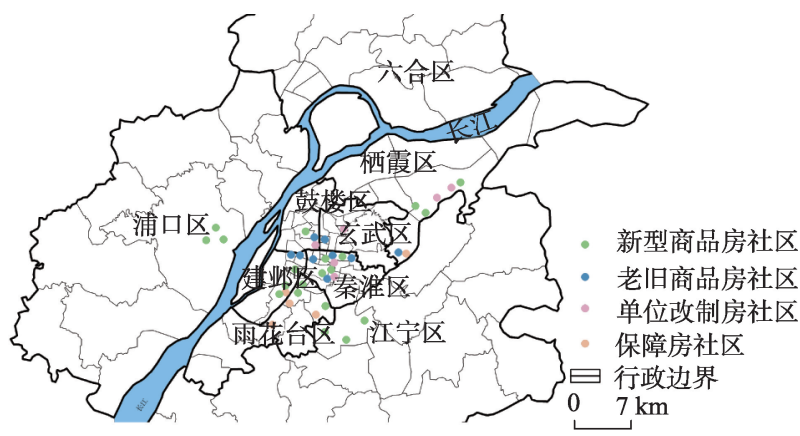

图 1 调研社区分布图

Fig.1 Location of the studied communities

表 1 样本统计性描述

Tab.1 Sample description

\begin{tabular}{|c|c|c|c|c|c|}
\hline 变量 & 样本量 & 比例\% & 变量 & 样本量 & 比例\% \\
\hline 性别 & & & 硕士及以上 & 177 & 14.08 \\
\hline 男性 & 611 & 48.61 & 家庭总计年收人 & & \\
\hline 女性 & 646 & 51.39 & $<5$ 万 & 186 & 14.80 \\
\hline 年龄 & & & $5 \sim 10$ 万 & 325 & 25.86 \\
\hline$\leqslant 24$ 岁 & 203 & 16.15 & 10 20万 & 430 & 34.21 \\
\hline 25 44 岁 & 747 & 59.43 & 20 30万 & 178 & 14.16 \\
\hline 45 59 岁 & 179 & 14.24 & $>30$ 万 & 117 & 9.31 \\
\hline$\geqslant 60$ 岁 & 129 & 10.26 & 社区类型 & & \\
\hline 受教育水平 & & & 新型商品房社区 & 617 & 49.09 \\
\hline 小学及以下 & 25 & 1.99 & 老旧商品房社区 & 197 & 15.67 \\
\hline 初中 & 135 & 10.74 & 单位改制房社区 & 167 & 13.29 \\
\hline 高中/中专 & 208 & 16.55 & 保障房社区 & 276 & 21.96 \\
\hline 大专/本科 & 708 & 56.32 & & & \\
\hline
\end{tabular}


(25 岁以下为参照项)、受教育程度(小学及以下为参 照项)、家庭年收人、社区类型(老旧型商品房社区为 参照项)。各变量的测度均使用李克特五级量表 (Likert Scale)进行评分, 具体测度问题见附录。社 区参与度的测度模型如下:

$$
\begin{gathered}
S=a+b+c \\
a=f\left(a_{1}+a_{2}+a_{3}+a_{4}\right) \\
b=f\left(b_{1}+b_{2}+b_{3}+b_{4}\right) \\
c=f\left(c_{1}+c_{2}+c_{3}+c_{4}\right)
\end{gathered}
$$

式中: $S$ 为社区参与度, $a$ 为信息查找强度, $b$ 为活动 参与度, $c$ 为组织参与度, $a_{1} 、 a_{2} 、 a_{3} 、 a_{4}$ 分别为事务型 活动、志愿型活动、文教型活动、休闲型活动信息查 找频次; $b_{1} 、 b_{2} 、 b_{3} 、 b_{4}$ 分别为事务型活动、志愿型活 动、文教型活动、休闲型活动参与频次; $c_{1} 、 c_{2} 、 c_{3} 、 c_{4}$ 分别为事务型社区组织、志愿型社区组织、文教型 社区组织、休闲型社区组织参与个数。

第二步, 通过因子分析计算社区参与度。结合 问卷数据, 本文对社区参与 “查找信息一参与活动 一参与组织” 3 个层面共 10 个指标(表 2)进行因子分 析, $\mathrm{KMO}$ 和 Bartlett 的检验中 $K M O$ 值为 0.887 , 通过 检验标准, 数据适宜作因子分析, 进一步计算出社 区参与的综合得分, 即社区参与度。结合样本属 性, 对南京市城市居民的社区网络的使用情况及社 区线上和线下参与的特征进行分析。

第三步,构建多元线性回归模型, 从 “媒介类 型”和“用途类型”2 个维度对社区网络的使用对社

\begin{tabular}{|c|c|c|}
\hline \multicolumn{3}{|r|}{ network usage } \\
\hline 一级变量 & 二级变量 & 具体测度问题 \\
\hline \multirow{9}{*}{ 社区参与度 } & & 查找社区文化教育类活动的频率 \\
\hline & & 查找社区娱乐休闲类活动的频率 \\
\hline & 信息查找 & 查找社区志愿/公益类活动的频率 \\
\hline & & 查找社区公共事务类活动的频率 \\
\hline & & 参与文化教育类活动的频率 \\
\hline & & 参与娱乐休闲类活动的频率 \\
\hline & 参与活动 & 参与社区志愿/公益类活动的频率 \\
\hline & & 参与社区公共事务类活动的频率 \\
\hline & 社区组织 & $\begin{array}{l}\text { 参与网上社区组织的数量(如社区 } \mathrm{QQ} \\
\text { 群、社区微信群等) } \\
\text { 参与过的线下社区组织的数量 }\end{array}$ \\
\hline
\end{tabular}
区参与的影响进行分析。模型同时也关注社区网

表 2 社区参与度测度指标体系

Tab.2 Measurement index system of community
络的使用、社区组织信任度、邻里联系、社区类型和 个人社会经济属性等因素的作用。此外,对全模型 的共线性进行检验, 发现所有变量的 VIF 均小于 5 , 因此可认为模型不存在明显的共线性。

\section{3 社区网络与社区参与特征}

\section{1 社区网络使用特征}

在调研的 1260 个样本中, 共有 902 个居民有社 区网络的使用经历, 占 $71.6 \%$, 使用者以 45 岁以下 的青年群体为主, 而 45 岁以上的中老年用户仅占 $23.2 \%$; 社区网络的使用强度总体平均值为 2.12 , 处 于“偶尔使用”的强度水平。具体分布特征如表 3 所 示, 社区网络的使用强度随年龄的上升显著下降, 随着受教育水平的上升整体增强,使用强度最高的 群体为 25 岁以下以及本科/大专学历的居民。

分媒介类型来看 (表 4 ), 居民使用度最高的是社 区 QQ 群/微信群, 其次为社区公众号/APP, 使用度 最低的为社区网站/社区论坛; 在不同的社区类型 中, 居民对社区网络的使用强度有较大差异, 其中 新型商品房社区的使用强度最高, 其次为老旧商品 房,使用强度最低的为单位型社区; 另外,不同类型 社区的居民对于社区网络媒介类型的选择有所不 同,新型商品房中的社区公众号/APP 的使用度高于

表3 社区网络的使用强度分布

Tab.3 Usage intensity of community networks

\begin{tabular}{ll|lc}
\hline \multicolumn{1}{c|}{ 年龄 } & 社区网络使用强度 & 受教育水平 & 社区网络使用强度 \\
\hline 25岁以下 & 2.61 & 初中及以下 & 1.60 \\
$25 ~ 45$ & 2.36 & 高中 & 2.12 \\
$45 ~ 60$ & 1.89 & 本科/大专 & 2.42 \\
60 岁以上 & 1.62 & 研究生及以上 & 2.34 \\
平均值 & 2.12 & 平均值 & 2.12 \\
\hline
\end{tabular}

注: 1 5 分分别代表从不、偶尔、一般、较多、非常频繁。

表4 社区网络媒介类型使用特征分布

Tab.4 Usage type of community networks

\begin{tabular}{lcccc}
\hline \multicolumn{1}{c}{ 社区类型 } & $\begin{array}{c}\text { 社区 } \mathrm{QQ} \text { 群/ } \\
\text { 微信群 }\end{array}$ & $\begin{array}{c}\text { 社区网站/ } \\
\text { 论坛 }\end{array}$ & $\begin{array}{c}\text { 社区公众号/ } \\
\text { APP }\end{array}$ & 总体 \\
\hline 新型商品房社区 & 2.75 & 2.26 & 2.52 & 2.51 \\
老旧商品房社区 & 2.41 & 2.06 & 2.01 & 2.16 \\
单位型社区 & 1.99 & 1.79 & 1.75 & 1.84 \\
保障房社区 & 2.10 & 1.92 & 1.88 & 1.97 \\
总体 & 2.32 & 2.01 & 2.04 & 2.12 \\
\hline
\end{tabular}

注: 1 5 分分别代表从不、偶尔、一般、较多、非常频繁。 
社区网站/论坛, 而老旧商品房、单位改制型社区和 保障房社区的居民对于社区公众号/APP 的使用度 要低于社区网站/论坛。因此可以看出, 新型商品房 社区的社区信息化水平相对更高, 居民对于社区公 众号/APP等更为高端的社区平台的可获性更高。

\section{2 基于“ $\mathrm{O} 2 \mathrm{O}$ ”的社区参与特征}

通过因子分析计算出各样本社区参与度的综 合得分, 并对结果进行标准化, 将标准化后的社区 参与度平均划分为极高 $(0.8 \sim 1.0)$ 、较高 $(0.6 \sim 0.8)$ 、一 般(0.4 0.6)、较低(0.2 0.4) 和极低( $0.0 \sim 0.2)$ 五级, 如 表 5 。首先, 总体来看, 南京市居民社区参与水平仅 有 $9.4 \%$ 为极高和较高水平, 大部分居民的参与水平 为较低 $(0.466)$ 和一般 $(0.246)$, 总体社区参与度平均 值仅为 0.35 ,处于 “较低” 水平。其次, 4 种类型社区 中居民的参与水平都处于 “较低” 水平, 但不同社区 之间仍然存在一定的差异,其中单位大院型社区的 居民社区参与水平为 4 种类型社区中最高, 社区参 与度平均值为 0.37 ; 其次为新型商品房社区和保障 房社区, 社区参与度平均值为 0.35 ; 老旧商品房社 区的居民社区参与水平最低, 社区参与度平均值为 0.33 。

按照社区参与度的分级对社区网络的使用情 况进行统计(表 6), 可以发现随着社区网络使用强度 和使用率的递增, 社区参与度呈显著的上升趋势, 在参与度 “极高” 等级的社区居民群体中, 有 $90 \%$ 的 居民使用社区网络, 此群体的社区网络平均使用强 度为 3.53 , 处于一般至较高层级。遵循和线下社区 参与一致的分类口径, 将社区网络的主要用途分为 文化教育、娱乐休闲、志愿公益、社区公共事务四大 类, 对社区网络的用途分布和线下参与特征分布进

表 5 不同类型社区的社区参与度分布

Tab.5 Levels of community participation

\begin{tabular}{lrrrrrc}
\hline \multicolumn{1}{c}{ 参与度 } & \multicolumn{7}{c}{ 极高 $/ \%$ 较高 $/ \%$ 一般 $/ \%$ 较低 $/ \%$ 极低 $/ \%$} & 平均值 \\
\hline 新型商品房社区 & 3.2 & 5.7 & 20.6 & 51.9 & 18.7 & 0.35 \\
老旧商品房社区 & 1.2 & 7.2 & 22.9 & 48.2 & 20.5 & 0.33 \\
单位型社区 & 1.2 & 10.2 & 28.7 & 40.7 & 19.2 & 0.37 \\
保障房社区 & 2.2 & 6.9 & 26.1 & 45.7 & 19.2 & 0.35 \\
总体 & 2.0 & 7.5 & 24.6 & 46.6 & 19.4 & 0.35 \\
\hline
\end{tabular}

表 6 社区参与度与社区网络使用特征

Tab.6 Community participation and characteristics of

community network use

\begin{tabular}{cccccc}
\hline 社区参与度 & 极高 & 较高 & 一般 & 较低 & 低 \\
\hline 社区网络使用强度 & 3.53 & 2.58 & 2.28 & 2.24 & 1.96 \\
社区网络使用率 $/ \%$ & 90.0 & 72.9 & 72.8 & 68.9 & 58.4 \\
\hline
\end{tabular}

行对比分析。均值统计结果如图 2 所示, 线下参与 中娱乐休闲类参与强度最高, 其次为文化教育和志 愿公益, 公共事务类参与强度最低, 说明现阶段南 京城市居民社区参与中非事务性参与高于事务性 参与, 居民的社区参与以个人需求为主导; 在社区 网络的使用方面, 4 种类型的用途分布和线下参与 大体一致,休闲娱乐类用途使用强度依然最大, 公 共事务类强度最低,但 4 种类型之间的差距减小,说 明社区网络的使用可在一定程度上促进社区参与 的均衡发展。

\section{4 社区参与的影响因素}

\section{1 模型与变量}

通过上文中社区参与的特征分析可以看出社 区网络的使用等方面的差异性。为进一步探究社 区参与各影响因素的综合作用, 以社区参与度为因 变量,从“类型”和“用途”两个维度考察社区网络使 用对社区参与的影响作用,分别建立多元线性回归 模型, 结合文献综述中相关研究成果(Chavis et al, 1990; Bimber, 1998; Carroll, Reese, 2003; Kavanaugh, 2005b; 杨敏, 2007; Anderson, 2009; Xu et al, 2010), 综合考虑社区归属感、社区组织信任度、个 人社会经济属性等内在影响因子, 以及邻里联系、 社区类型等外界影响因子,对社区参与的影响因素 进行回归分析。数据通过残差正态性和方差齐性、 独立性检验,模型的结构如下:

$$
\begin{aligned}
S= & \beta_{0}+\beta_{1} \cdot X_{1}+\beta_{2} \cdot X_{2}+\beta_{3} \cdot X_{3}+\beta_{4} \cdot X_{4}+ \\
& \beta_{5} \cdot X_{5}+\beta_{6} \cdot X_{6}
\end{aligned}
$$

式中: $S$ 为社区参与度, $X_{1}$ 为社区网络的类型, $X_{2}$ 为社区网络的用途, $X_{3}$ 为社区组织信任度, $X_{4}$ 为 邻里联系, $X_{5}$ 为社区归属感, $X_{6}$ 为个人社会经济 属性, $\beta_{0} \cdots \beta_{6}$ 为各项系数。

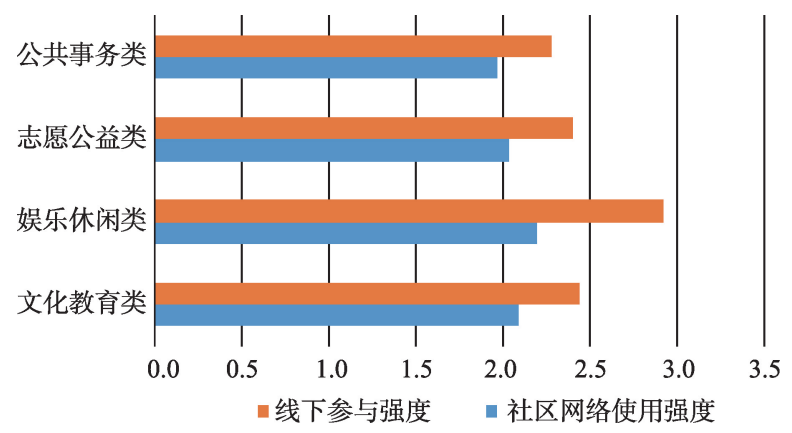

图 2 线上线下社区活动类型的参与率分布

Fig.2 Participation rate of online and offline community participation types 


\section{2 结果分析}

社区参与的 3 个层级影响因素变量选定后, 在 SPSS statistics19.0 中分别构造模型, 进行多元回归 分析, 结果如表 7 所示, 两组模型都表明社区网络的 使用对社区参与有显著影响。

(1) 社区网络的使用对社区参与有一定促进作 用。在社区网络的不同媒介类型中,社区网站、社
区 QQ 群/微信群、社区公众号/APP等网络平台三者 对于社区参与都有一定的正向影响, 其中社区公众 号/APP 的促进作用最为显著,标准回归系数 0.197 ; 其次为社区 $\mathrm{QQ}$ 群/微信群, 回归系数为 0.061 ; 社区 网站的正向影响作用相对微弱, 回归系数值也最 小, 为 0.059 。在移动互联网的应用日益广泛和深 人的背景下, 社区 $\mathrm{QQ}$ 群/微信群、微信公众号/APP

表 7 社区参与影响因素的多元回归模型

Tab.7 Results of multiple regression model of influencing factors of community participation

\begin{tabular}{|c|c|c|c|c|c|c|}
\hline \multirow{2}{*}{ 自变量 } & \multicolumn{3}{|c|}{ 模型一 } & \multicolumn{3}{|c|}{ 模型二 } \\
\hline & 系数 $(B)$ & 标准误差 & Beta & 系数 $(B)$ & 标准误差 & Beta \\
\hline (常量) & -0.225 & 0.039 & & -0.174 & 0.034 & \\
\hline \multicolumn{7}{|l|}{ 社区网络的类型 } \\
\hline 社区网站/论坛 & $0.010^{*}$ & 0.005 & 0.059 & & & \\
\hline 社区 QQ群/微信群 & $0.024^{* *}$ & 0.010 & 0.061 & & & \\
\hline 社区公众号/APP & $0.030^{* * * *}$ & 0.005 & 0.197 & & & \\
\hline \multicolumn{7}{|l|}{ 社区网络的用途 } \\
\hline 文化教育类 & & & & $0.016^{* *}$ & 0.007 & 0.098 \\
\hline 休闲娱乐类 & & & & 0.009 & 0.006 & 0.060 \\
\hline 公益志愿类 & & & & $0.023^{* * *}$ & 0.007 & 0.132 \\
\hline 公共事务类 & & & & $0.035^{* * *}$ & 0.007 & 0.193 \\
\hline \multicolumn{7}{|l|}{ 社区组织信任度 } \\
\hline 社区居委会 & $0.035^{* * * *}$ & 0.006 & 0.163 & $0.032^{* * * *}$ & 0.006 & 0.151 \\
\hline 物业管理部门 & 0.000 & 0.006 & 0.001 & -0.005 & 0.005 & -0.028 \\
\hline 业主委员会 & 0.000 & 0.006 & -0.001 & 0.002 & 0.005 & 0.012 \\
\hline 社区自发团体 & $0.018^{* * * *}$ & 0.006 & 0.091 & $0.011^{* *}$ & 0.005 & 0.056 \\
\hline 邻里联系 & $0.015^{* * *}$ & 0.002 & 0.214 & $0.012^{* * * *}$ & 0.002 & 0.172 \\
\hline 社区归属感 & $0.016^{* * * *}$ & 0.002 & 0.183 & $0.011^{* * * *}$ & 0.002 & 0.135 \\
\hline \multicolumn{7}{|l|}{ 性别(女性为参照) } \\
\hline 男性 & $0.027^{* * * *}$ & 0.009 & 0.071 & $0.027^{* * * *}$ & 0.008 & 0.071 \\
\hline \multicolumn{7}{|l|}{ 年龄(小于 25 岁为参照) } \\
\hline $25 \sim 44$ & $-0.021^{* *}$ & 0.011 & -0.056 & $-0.025^{* * *}$ & 0.010 & -0.067 \\
\hline $45 \sim 59$ & -0.005 & 0.010 & -0.013 & -0.003 & 0.009 & -0.008 \\
\hline 60 以上 & $0.035^{* * *}$ & 0.013 & 0.074 & $0.026^{* *}$ & 0.012 & 0.055 \\
\hline \multicolumn{7}{|c|}{ 受教育水平(初中及以下为参照) } \\
\hline 高中、中专 & 0.015 & 0.017 & 0.029 & -0.010 & 0.016 & -0.019 \\
\hline 大专、本科 & -0.016 & 0.016 & -0.042 & -0.025 & 0.015 & -0.066 \\
\hline 硕士及以上 & -0.013 & 0.021 & -0.025 & -0.028 & 0.019 & -0.053 \\
\hline 家庭总计年收人 & $-0.005^{*}$ & 0.003 & -0.051 & $-0.004^{*}$ & 0.002 & -0.044 \\
\hline \multicolumn{7}{|c|}{ 社区类型(老旧型商品房社区为参照) } \\
\hline 新型商品房社区 & $0.052^{* * * *}$ & 0.020 & 0.137 & $0.043^{* *}$ & 0.017 & 0.115 \\
\hline 单位改制型社区 & $0.057^{* * *}$ & 0.022 & 0.106 & $0.072^{* * *}$ & 0.019 & 0.132 \\
\hline 保障房社区 & $0.050^{* *}$ & 0.021 & 0.112 & $0.035^{*}$ & 0.018 & 0.078 \\
\hline$R^{2}$ & 0.330 & & & 0.429 & & \\
\hline 调整 $R^{2}$ & 0.318 & & & 0.419 & & \\
\hline
\end{tabular}

注:因变量:社区参与度; $* * * * *$ *分别表示在 $0.01 、 0.05 、 0.1$ 水平上显著。 
等移动互联网络平台可更为便捷和深人地使居民 建立与社区的联系, 从而更为有效地促进社区参与 的发生, 社区网站/论坛等媒介也能为社区参与提供 一定的信息, 但是由于其即时性和渗透性较弱, 对 于社区参与和社区活动的促进作用相比于社区 $Q Q$ 群/微信群、社区公众号/APP来说相对有限。

(2) 社区网络的不同用途对社区参与的促进作 用不同。具体来看, 社区文化教育、社区公益志愿 以及社区公共事务类的社区网络应用与社区参与 有着极其显著的正相关关系, 标准回归系数分别为 $0.098 、 0.132$ 和 0.193 , 说明在文化教育类、公益志愿 类和公共事务类参与方面, 社区网络的促进作用较 显著, 尤其是社区公益和事务型参与类型, 线上的 关注更有可能转为线下参与。主要原因可能是休 闲娱乐类活动的参与相关信息的获知门槛较低, 线 下交流即可满足大多数参与需求, 且休闲娱乐类社 区参与主体以中老年为主, 对于社区网络的使用度 也较低, 因而社区网络在这类参与中所起到的作用 较小; 而社区文化教育、社区公益志愿活动和社区 公共事务类活动的线下信息获取往往相对不足,社 区网络则在信息渠道方面起到一定程度的弥补作 用, 从而促进此类社区活动的参与。因而, 社区网 络的使用可以在某种程度上促进社区参与从娱乐 类参与向较高层次公益志愿和公共事务类的参与 活动演进, 有助于社区参与深度的提升。

(3) 社区组织信任度、邻里联系、社区归属感对 社区参与均有不同程度的影响。其中, 在社区组织 信任度指标中, 社区居委会和社区自发团体的信任 度对社区参与有着显著的正向影响作用, 但物业组 织和业主委员会的影响并不显著; 邻里联系、社区 归属感对居民社区参与有较为显著的正向影响。 主要原因为现阶段大多数物业公司的关注领域为 社区服务, 对社区活动方面的投人较少, 而业主委 员会在很多社区中处于 “形同虚设”的地位, 居民普 遍信任度不高, 在社区参与方面发挥的作用也极为 有限。相比之下, 大多数社区活动为居民自发组织 和参与, 因而社区居民自发团体对于社区参与的促 进作用最为显著。同时, 社区参与作为一种集体活 动, 邻里联系是信息获取的重要渠道之一, 因此邻 里联系在社区参与中扮演着重要角色。社区归属 感较高的社区居民对于社区公共活动和事务的关 注度更高, 因而会更加乐于进行社区参与。

（4）居民的性别、年龄、受教育程度、家庭总计 年收人以及社区类型对居民的社区参与有一定的
影响。其中, 在性别因素方面, 男性相比于女性有 相对更高的社区参与度; 在年龄方面, 相比于 25 岁 以下的年轻人群体, 25 40 岁的青年人群体的社区 参与度更低,而 60 岁以上的老年人群体的社区参与 度最高; 受教育水平对居民的参与度无显著影响。 由于青年人群体往往要付出更多的时间和精力忙 于工作和家庭, 社区参与的时间成本相对更高, 而 老年人由于行动能力的减弱、空闲时间的相对充 足, 邻里互动和对社区生活的关注都相对更高。在 社区类型方面, 相比于老旧商品房社区, 新型商品 房社区、单位型社区和保障房社区居民的社区参与 都有显著的正相关关系。老旧商品房社区的社区 参与受到建成环境、公共空间、社区网络建设不足 等多种限制, 社区参与水平较低; 单位型社区由于 邻里间联系更为密切, 社区信任感也较强, 因而总 体参与水平较高。

\section{5 结论与讨论}

本文基于国内社区参与和智慧社区领域社区 网络相关研究的不足, 结合中国社区网络实践现状 及特点, 对中国城市社区居民中社区网络的使用特 征进行描述分析,并从“媒介”和“用途” 2 个维度对 社区网络的使用对社区参与的影响机制进行深人 探究。研究的主要结论为:

(1) 南京市城市居民社区网络使用总体呈现 “高普及、低强度、新社区”的特征, 社区网络的普及 度较高, 71.6\%的被调研者(18岁以上社区居民)有 社区网络的使用经历, 然而平均使用强度并不高, 仅处于 “偶尔使用”水平; 在不同的社区类型中居民 的社区网络使用强度不同,其中新型商品房社区的 使用强度最高, 单位型社区使用强度最低, 这与社 区的信息化水平以及人群特征相关, 新型社区的信 息化水平往往相对较高,而单位型社区居民彼此更 为熟络, 线下交流在某种程度上替代了使用社区网 络的必要性。

(2) 在社区网络类型的使用方面, 呈现 “以移动 互联为主” 的特征。居民使用度最高的是社区 $\mathrm{QQ}$ 群/微信群, 其次为社区公众号/APP, 使用度最低的 为社区网站/社区论坛。由此可以看出, 当下中国城 市居民社区生活中, 尤其是新型商品房社区, 社区 网络的应用和普及已经初见成效, 其中 QQ 群/微信 群等线上自发组织的普及率已达到相对较高水平, 但在应用深度和用户吸引力方面还有待进一步 
提升。

(3) 在社区“O2O”方面, 与西方研究结果相互 印证。中国城市居民社区网络的使用对线下社区 参与也有显著促进作用, 且社区网络的使用有助于 社区参与深度的提升, 促进非事务型社区参与向事 务型社区参与的演进。随着社区网络使用率和使 用强度的递增, 居民的社区参与度总体呈现显著上 升趋势; 在社区网络的具体用途中, 文化教育、公益 志愿、公共事务类用途的线上线下互动效果更佳, 说明社区网络的使用可以在一定程度上促进社区 参与的均衡发展和深度提升。另外, 本文结合中国 社区网络发展和应用现状, 进一步对不同类型社区 网络的影响进行深人探究。研究表明, 不同类型的 社区网络对社区参与的促进作用不同, 社区公众号/ APP 类的社区网络类型对社区参与的促进作用最 为显著, 其次为社区 $\mathrm{QQ}$ 群/微信群, 社区网站/论坛 对社区参与的促进作用最为微弱, 说明移动互联网 在中国城市居民社区生活中的渗透更为深人。

(4) 在社区网络影响因素之外, 城市居民社区 参与也在一定程度上受到邻里联系、社区归属感以 及个人社会经济特征的影响, 且相比于社区网络的 作用, 社区组织、邻里联系和社区归属感等传统因 素在社区参与中的关键作用仍然不可替代。值得 关注的是, 与西方国家有所不同,在社区自组织团 体之外, 居委会在社区参与中仍扮演着比较关键的 作用, 这与中国城市社区作为国家治理单元的性质 密切相关。

为此, 在智慧社区的建设中应重视并加强社区 网络的构建, 重点推广微信公众号/APP 以及社区 QQ群/微信群等社区网络平台的建设, 并通过社区 网络提供更多的文化教育、公益志愿以及公共事务 类的社区活动信息, 促使更多居民关注并参与到社 区公共活动和事务中。同时也应关注人群和社区 的异质性, 提升社区活动的多样性。另外, 结合中 国社区组织和社区治理的特点, 社区居委会应充分 发挥在社区参与方面的关键作用, 鼓励和扶持社区 自发团体的成立并监督其规范化运作; 并且, 居委 会应充分利用社区网络这一沟通媒介, 在作为国家 治理空间的社区与作为日常生活空间的社区之间 建立连接机制, 使两者形成相对平衡的关系, 为社 区的发育创造坚实的基础(吴光芸, 2007)。今后社 区信息化建设需要更多地弥补对社区网络及社区 参与关注的不足, 积极引导居民通过线上重新回到
线下, 通过社区活动的参与融人社区公共生活, 通 过面对面的交流产生更多的价值, 从而带动社区活 力的激发。

本文也存在一些局限, 线性回归模型只能粗略 地表明社区网络与社区参与之间的相关性, 而社区 网络对社区参与更加具体的作用机制、自发性社区 网络的构建和官方主导建设的社区网络的作用与 效果的对比等方面, 都有待于今后作进一步的深人 探索。

\section{参考文献(References)}

柴彦威, 郭文伯. 2015. 中国城市社区管理与服务的智慧化 路径 [J]. 地理科学进展, 34(4): 466-472. [Chai Y W, Guo W B. 2015. Smart management and service of communities in Chinese cities[J]. Progress in Geography, 34(4): 466472.]

陈卉, 甄峰. 2016. 信息通讯技术对老年人的社区满意度影 响路径: 以南京市锁金社区为例 [J]. 地理科学进展, 35 (9): 1167-1176. [Chen H, Zhen F. 2016. Impact of ICT on community satisfaction among elderly: The case of Suojin Community in Nanjing City[J]. Progress in Geography, 35 (9): 1167-1176.]

陈雅丽. 2002. 城市社区发展中的居民参与问题[J]. 科学·经 济・社会, 20(3): 55-58. [Chen Y L. 2002. Chengshi shequ fazhan zhong de jumin canyu wenti[J]. Science, Economy, Society, 20(3): 55-58.]

陈振华. 2004. 利益、认同与制度供给: 居民社区参与的影响 因素研究[D]. 北京: 清华大学. [Chen Z H. 2004. Interest, identity and institutional supply: A study on the influence factors of community participation[D]. Beijing, China: Tsinghua University.]

黄荣贵, 桂勇. 2011. 集体性社会资本对社区参与的影响: 基 于多层次数据的分析 [J]. 社会, 31(6): 1-21. [Huang R G, Gui Y. 2011. Collective social capital and its effect on community participation: A multilevel analysis[J]. Chinese Journal of Sociology, 31(6): 1-21.]

申悦, 柴彦威, 马修军. 2014. 人本导向的智慧社区的概念、 模式与架构 [J]. 现代城市研究, (10): 13-17, 24. [Shen Y, Chai Y W, Ma X J. 2014. Concept model and framework of human-oriented smart community[J]. Modern Urban Research, (10): 13-17, 24.]

孙璐. 2006. 利益、认同、制度安排: 论城市居民社区参与的 影响因素[J]. 云南社会科学, (5): 70-73. [Sun L. 2006. Interests, identification and system arrangement: An analysis on influential factors in participation of urbanite's community[J]. Social Sciences in Yunnan, (5): 70-73.]

滕尼斯. 1999. 共同体与社会[M]. 林荣远, 译 北京: 商务印书 
馆. [Tönnies F. 1999. Gemeinschaft und gesellschaft[M]. Lin R Y, Trans.. Beijing, China: Commercial Press.]

王刚, 罗峰. 1999. 社区参与: 社会进步和政治发展的新驱动 力和生长点: 以五里桥街道为案例的研究报告 [J]. 浙江 学刊, (2): 72-75. [Wang G, Luo F. 1999. Shequ canyu: Shehui jinbu he zhengzhi fazhan de xinqudongli he shengzhangdian: Yi Wuliqiao Jiedao wei anli de yanjiu baogao [J]. Zhejiang Academic Journal, (2): 72-75.]

吴光芸. 2007. 利益相关者合作逻辑下的我国城市社区治理 结构 [J]. 城市发展研究, 14(1): 82-86. [Wu G Y. 2007. Community governance structures in the logic of stakeholders cooperation[J]. Urban Studies, 14(1): 82-86.]

吴光芸, 杨龙. 2006. 社会资本视角下的社区治理 [J]. 城市发 展研究, 13(4): 25-29. [Wu G Y, Yang L. 2006. The community governance in the view of social capital[J]. Urban Studies, 13(4): 25-29.]

吴晓林, 郝丽娜. 2015. “社区复兴运动” 以来国外社区治理 研究的理论考察 [J]. 政治学研究, (1): 47-58. [Wu X L, Hao L N. 2015. "Shequ fuxing yundong" yilai guowai shequ zhili yanjiu de lilun kaocha[J]. Cass Journal of Political Science, (1): 47-58.]

杨敏. 2007. 作为国家治理单元的社区: 对城市社区建设运 动过程中居民社区参与和社区认知的个案研究 [J]. 社会 学研究, (4): 137-164. [Yang M. 2007. Community as state governance unit: A case study on residents community participation and cognition in the process of community building campaign[J]. Sociological Studies, (4): 137-164.]

叶南客. 2001. 中国城市居民社区参与的历程与体制创新 [J]. 江海学刊, (5): 34-41. [Ye N K. 2001. The process and system innovation of community participation in urban China[J]. Jianghai Academic Journal, (5): 34-41.]

张庭伟. 1999. 社会资本、社区规划及公众参与 $[\mathrm{J}]$. 城市规 划, 23(10): 23-26, 30. [Zhang T W. 1999. Social capital: Community planning and public participation[J]. City Planning Review, 23(10): 23-26, 30.]

张卫. 2001. 社区参与: 社区建设与发展的推动力: 对南京市 锁金村社区的个案分析 [J]. 社会, (1): 12-14. [Zhang W. 2001. Shequ canyu: Shequ jianshe yu fazhan de tuidongli: Dui Nanjingshi Suojincun Shequ de ge'an fenxi[J]. Chinese Journal of Sociology, (1): 12-14.]

甄峰, 席广亮, 秦萧. 2015. 基于地理视角的智慧城市规划与 建设的理论思考 [J]. 地理科学进展, 34(4): 402-409. [Zhen F, Xi G L, Qin X. 2015. Smart city planning and construction based on geographic perspectives: Some theoretical thinking $[\mathrm{J}]$. Progress in Geography, 34(4): 402409.]

Almond G A, Verba S. 1963. The civic culture: Political attitudes and democracy in five nations[M]. Princeton, NJ:
Princeton University Press.

Anderson M R. 2009. Beyond membership: A sense of community and political behavior[J]. Political Behavior, 31(4): 603-627.

Bimber B. 1998. The internet and political transformation: Populism, community, and accelerated pluralism[J]. Polity, 31(1), 133-160.

Brodsky A E, O'Campo P J, Aronson R E. 1999. PSOC in community context: Multi-level correlates of a measure of psychological sense of community in low- income, urban neighborhoods $[\mathrm{J}]$. Journal of Community Psychology, 27 (6): 659-679.

Carroll J M, Reese D D. 2003. Community collective efficacy: Structure and consequences of perceived capacities in the Blacksburg Electronic Village[C]//Proceedings of the 36th Annual Hawaii International Conference on System Sciences. Big Island, HI: IEEE: 10.

Carroll J M, Rosson M B. 2003. A trajectory for community networks special issue: ICTs and community networking [J]. The Information Society, 19(5): 381-393.

Chavis D M, Wandersman A. 1990. Sense of community in the urban environment: A catalyst for participation and community development[J]. American Journal of Community Psychology, 18(1): 55-81.

Dimaggio P, Hargittai E, Celeste C, et al. 2003. From unequal access to differentiated use: A literature review and agenda for research on digital inequality[R]. Working Paper. Princeton, NJ: Robertson Hall: 355-400.

Hampton K N. 2003. Grieving for a Lost Network: Collective action in a wired suburb special issue: ICTs and community networking $[\mathrm{J}]$. The Information Society: An International Journal, 19(5): 417-428.

Kavanaugh A, Carroll J M, Rosson M B, et al. 2005a. Community networks: Where offline communities meet online[J]. Journal of Computer- Mediated Communication, 10(4), doi: 10.1111/j.1083-6101.2005.tb00266.x.

Kavanaugh A, Carroll J M, Rosson M B, et al. 2005b. Participating in civil society: The case of networked communities [J]. Interacting with Computers, 17(1): 9-33.

Kling R, Iacono S. 1989. The institutional character of computerized information systems[J]. Information Technology \& People, 5(1): 7-28.

Milbrath L W. 1977. Political participation: How and why do people get involved in politics[M]. 2nd ed. Chicago, IL: Rand McNally.

Putnam R D. 1995. Tuning in, tuning out: The strange disappearance of social capital in America[J]. PS: Political Science \& Politics, 28(4): 664-683. 
Putnam R D. 2000. Bowling alone: The collapse and revival of American community[M]. New York, Simon \& Schuster.

Schuler D. 1996. New community networks: Wired for change

[M]. New York, Addison Wesley Publishing.

Wellman B, Haase A Q, Witte J, et al. 2001. Does the Internet increase, decrease, or supplement social capital? Social net- works, participation, and community commitment[J]. American Behavioral Scientist, 45(3): 436-455.

Xu Q W, Perkins D, Chow J C C. 2010. Sense of community, neighboring, and social capital as predictors of local political participation in China[J]. American Journal of Community Psychology, 45(3-4): 259-271.

\title{
Use of community networks and its impact on community participation: A case study of Nanjing City
}

\author{
CHANG Enyu ${ }^{1}$, ZHEN Feng ${ }^{1,2^{*}}$, SUN Chen ${ }^{1}$ \\ (1. School of Architecture and Urban Planning, Nanjing University, Nanjing 210093, China; \\ 2. Human Geography Research Center of Nanjing University, Nanjing University, Nanjing 210093, China)
}

\begin{abstract}
In the 1970s, the United States launched a large number of practices and research focusing on "community network" in community informatics area. In recent years, with the rapid penetrating of information and communication technology in the daily life of community residents, virtual network platforms based on the community entity also appear in large numbers in urban communities of China, which brings an opportunity to improve community participation of urban residents. However, research by Chinese scholars rarely concerned with community networks, especially that quantitative research in this area has been few. Under this background and taking into consideration the practice of community networks in China, this study analyzed the characteristics of community network use and community participation of residents in Nanjing City based on a sample survey. Furthermore, the study used multiple- linear regression method to investigate the factors influencing community participation. The survey was conducted in 35 communities of Nanjing City by a research group at Nanjing University. The results show that overall community network use was characterized by high popularity and low intensity. Although the average coverage of community network use of Nanjing residents was high, the intensity of use only remained at the "occasional use" level. Most users were from newly built communities. As for the usage type of community networks, "mobile Internet" was most popular among the residents, and community QQ/WeChat groups showed the highest use intensity, followed by community Official Accounts/APPs, and community websites/community forums had the lowest use intensity. With regard to community "O2O", community participation was significantly promoted by using community networks. The use of community networks will enhance the depth of participation by promoting transaction type community participation. This article proposes that smart community development should compensate for the lack of attention to community networks and community involvement, and facilitate a return to community public life through participation in community activities that increases the vitality of communities.
\end{abstract}

Key words: urban community; community participation; community network; smart community; community "O2O”; Nanjing City 